\title{
THE POWER MEAN AND THE LOGARITHMIC MEAN
}

\section{CHRISTOPHER OLUTUNDE IMORU}

\author{
Department of Mathematics \\ University of Ife \\ Ile-Ife \\ Oyo State \\ NIGERIA
}

(Received July 3, 1979

and in revised form, March 24, 1980)

ABSTRACT. In a very interesting and recent note, Tung-Po Lin [1] obtained the least value $q$ and the greatest value $p$ such that

$$
M_{p}<\mathrm{L}<M_{q}
$$

is valid for all distinct positive numbers $x$ and $y$ where

$$
M_{s}=\left(\frac{x^{s}+y^{s}}{2}\right)^{\frac{1}{s}} \text { and } L=\frac{x-y}{\operatorname{In} x-\operatorname{In} y}
$$

The object of this paper is to give a simpler proof than Lin's of a more general result. More precisely, the author obtained the classes of functions $f_{\alpha}$ and $h_{\alpha}, \alpha \varepsilon R$ such that

$$
\text { In } f_{\alpha}(t)-h_{\alpha}(t)\left[t^{1 / \alpha}+1\right]^{-\alpha}>0, t>1 .
$$

KEY WORDS AND PHRASES. Concave Functions, Space of Lebesque $\mu$-integrable functions.

1980 MATHEMATICS SUBJECT CLASSIFICATION CODES. Primary 26 A 86.

1. INTRODUCTION.

There is an extensive literature on the extensions and generalizations of inequalities involving the power mean and the logarithmic mean and their applica- 
tions [see 2-5]. Tung-Po Lin [1] considered the least value $p$ and the greatest value $q$ such that

$$
\mathrm{M}_{\mathrm{q}}<\mathrm{L}<\mathrm{M}_{\mathrm{p}}
$$

is valid for all distinct positive real numbers $x$ and $y$, where

$$
L=L(x, y)=\frac{x-y}{I n x-I n y} \text { and } M_{\alpha}=M_{\alpha}(x, y)=\left(\frac{x^{\alpha}+y^{\alpha}}{2}\right), \alpha \varepsilon R
$$

are respectively the logarithmic mean and the power mean of order $\alpha$ of two distinct positive numbers $x$ and $y$.

Indeed, Lin showed that $q=0$ and $p=\frac{1}{3}$ are the best possible values one can get in order that (1.1) may be satisfied for any distinct positive real numbers $x$ and $y$. This result is sharper than an earlier inequality due to Carlson [6], namely:

$$
M_{0}<L<M_{\frac{1}{2}} \text {, for any distinct } x, y>0 \text {. }
$$

As observed by Lin ([1], Theroems 3 and 4$)$, if $0<p<1 / 3$, there exist $x>0$ and $y>0$ such that $L(x, y)>M_{p}(x, y)$. On the other hand, there exist $x>0$ and $y>0$ such that $L(x, y)<M_{p}(x, y)$. In fact, if we suppose that $x>y>0$, which is perfectly in order due to the symmetry of $L(x, y)$ and $M_{p}(x, y)$ in $x$ and $y$, one can readily show that

$$
L(x, y)<M_{p}(x, y), o<p<\frac{1}{3}
$$

if $\frac{x}{y}$ is large enough. The reversed inequality holds for smaller values of $\frac{x}{y}$. For instance, numerical calculations with the aid of a computer easily show that the result (1.3) holds for $p=\frac{1}{4}, \frac{1}{5}, \ldots, \frac{1}{10}$ if $\frac{x}{y}$ is greater than (9.9) $4,(25.3799)^{5}$, $(56.6188)^{6},(119.7628)^{7},(246.8528)^{8},(501.9099)^{9}$ and $(1012.9460)^{10}$ respectively.

From these results, we can readily compute the set $\{(x, y) ; x>0$ and $y>0\}$ for which $M_{p}(x, y)<L(x, y), \frac{1}{4}>p>\frac{1}{10}$. For example, $M_{1}(x, y)<L(x, y)$ on the set $\left\{(x, y) ; \frac{x}{y}<(1012.9460)^{10}\right\}$. It is interesting to note that the above esti- 
mates show that $\frac{x}{y} \rightarrow \infty$ as $p \rightarrow o$, which is compatible with the left-hand side of inequality (1.1), namely:

$$
M_{0}(x, y)<L(x, y) \text { for distinct } x>y>0
$$

Furthermore, the critical value of $(x / y)^{1 / p}$ more than doubles from $p=1 /(4+n)$ to $p=1 /(5+n)$, where $n=0,1,2, \ldots . ., 5$. It is our conjecture that the critical values of $(x / y)^{1 / p}$ is more than double from $p=1 / n$ to $1 /(n+1), n=1, n,$. . .

The purpose of this note is to give a simpler proof than Carlson or Lin of a more general result. Indeed, we obtain in Section 2 the classes of functions $f_{\alpha}$ and $h_{\alpha}, \alpha \varepsilon R$, for which the function

$$
F_{\alpha}(t)=\operatorname{In} f_{\alpha}(t)-h_{\alpha}(t)\left(t^{1 / \alpha}+1\right)^{-\alpha}, t>1
$$

is strictly positive. This leads to the determination of a polynomial of degree $n$, $(n \geq 1)$ which is a lower bound of the function $H(t)=\left(\frac{t+1}{2}\right)^{n}$ In $t$. The comparison of these bounds for $\mathrm{n}=1,2, \ldots$ with the ones given by Carlson and Lin show that not only are our results more general in form, but they are somewhat sharper. We point out that Carlson's inequalities (1.2) are special cases of an inequality for convex and concave functions. Indeed, from the Jensen inequality for concave functions,

$$
\frac{\int_{1}^{t} f(x) d \mu(x)}{\int_{1}^{t} d \mu(x)}>\varphi^{-1}\left[\frac{\int_{1}^{t}\langle\{f(x)\} d \mu(x)}{\int_{1}^{t} d \mu(x)}\right]
$$

where is concave and $f \varepsilon L(R, d \mu)$, the space of Lebesque $\mu$ - integrable functions on $R$, we obtain, on setting $f(x)=\frac{1}{x}, \mu(x)=x$ and $\varphi(x)=x^{1 / p}, p>1$ or $\mathrm{p}<\mathrm{o}$, the inequality

$$
\frac{\operatorname{In} t}{t-1}>\left[\frac{p}{p-1} \frac{t^{1-1 / p}-1}{t-1}\right]^{p}, p>1 \text { or } p<0 .
$$

Since (1.4) is reversed if $\varphi$ is convex, inequality (1.5) is reversed if $0<\mathrm{p}<1$. Taking $\mathrm{p}=2$ in (1.5), we obtain the right-hand side of inequality $(1.2)$.

\section{MAIN RESULTS.}

We pointed out that Lin, with the aid of the Maclaurin series, showed that 
the best possible value of $p$ for which $L<M_{p}$ is $\frac{1}{3}$. A proof of this interesting result without the Maclaurin series was indicated in the paper [1] by Harley Flanders. Following Flander's approach, we obtain the following result.

Theorem 2.1: Let $F_{\alpha}(z)=\ln f_{\alpha}(z)-h_{\alpha}(z)(z+1)^{-\alpha}, \alpha \varepsilon R$, where $f_{\alpha}$ is an increasing positive function and $h_{\alpha}$ is differentiable in $R$. Suppose $f_{\alpha}(1)=1$ and $h_{\alpha}(1)=0$. Then $F_{\alpha}(z)>0$, for every $z>1$, provided

$$
(z+1)^{\alpha+1}-\frac{h_{\alpha}^{1}(z) f_{\alpha}(z)}{f_{\alpha}^{1}(z)}(z+1)+\alpha \frac{h_{\alpha}(z) f_{\alpha}(z)}{f_{\alpha}^{1}(z)}>0 .
$$

Proof: Computing the first derivative of $f_{\alpha}(z)$ yields

$$
F_{\alpha}^{1}(z)=\Delta_{\alpha}(z)\left[(z+1)^{\alpha+1}-G_{\alpha}(z)\right]
$$

where

$$
\Delta_{\alpha}(z)=\frac{f_{\alpha}^{1}(z)}{f_{\alpha}(z)(z+1)^{\alpha+1}}
$$

and

$$
G_{\alpha}(z)=\frac{h_{\alpha}^{1}(z) f_{\alpha}(z)}{f_{\alpha}^{1}(z)}(z+1)-\alpha \frac{h_{\alpha}(z) f_{\alpha}(z)}{f_{\alpha}^{1}(z)}
$$

Observe that $F_{\alpha}^{\prime}(z) \geqq 0$ if and only if $(z+1)^{\alpha+1}-G_{\alpha}(z) \geqq 0, z \geqq 1$. Since $F_{\alpha}(1)=0$ and $F_{\alpha}^{\prime}(z)>0$ wherever $z>1$, we conclude that $F_{\alpha}(z)>0$, for all $z>1$. This completes the proof of the theorem.

Corollary 2.2: For every $\beta_{k} \varepsilon R, 1<k<\alpha$, where $\alpha \varepsilon \mathbb{Z}_{+}$, the inequality

$$
\left.\frac{1}{\alpha \operatorname{In} z} \sum_{k=1}^{\alpha} \frac{\beta_{k}}{k}\left(z^{k}-1\right)<z+1\right)^{\alpha}, z>1,
$$

is valid provided

$$
\begin{gathered}
z^{\alpha+1}+\sum_{k=2}^{\alpha}\left[{ }^{\alpha+1} C_{k}+\frac{\beta_{k-1}}{k-1}-\left[\frac{\beta_{k-1+\beta}}{\alpha}\right]\right] z^{k} \\
+\left[\alpha+1+\frac{\beta_{1}}{\alpha}-\sum_{k=1}^{\alpha} \frac{\beta_{k}}{k}\right] z+1>0 .
\end{gathered}
$$


Proof: Let $h_{\alpha}(z)=\sum_{k=1}^{\alpha} \frac{\beta_{k}}{k}\left(z^{k}-1\right)$ and $f_{\alpha}(z)=z^{\alpha}$ in Theorem 2.1. Then the result follows at once.

Observe that for all $z>1$,

$$
\sum_{k=1}^{\alpha} \frac{\beta_{k}}{k}\left(z^{k}-1\right) \geqq 2^{\alpha}\left(z^{\alpha}-1\right), \text { provided }\left(\beta_{k}\right)_{1 \leqq k \leqq \alpha}
$$

satisfies the conditions

$$
\beta_{\alpha} \geqq \alpha 2^{\alpha} \text { and } \sum_{k=1}^{\alpha-1} \frac{\beta_{k}}{k}\left(z^{k}-1\right) \geqq 0
$$

The inequality (2.5) is strict unless $\beta_{\alpha}=\alpha 2^{\alpha}$ and $\beta_{k}=0,1<k<\alpha$. Consequently, we have

$$
\frac{2^{\alpha}\left(z^{\alpha}-1\right)}{\alpha \ln z} \leqq \frac{1}{\alpha \ln z} \sum_{k=1}^{\alpha} \frac{\beta_{k}}{k}\left(z^{k}-1\right)<(z+1)^{\alpha}, \alpha \mathbb{Z}_{7}
$$

wherever (2.4) and (2.6) are valid.

Hence for all $z>1$, the polynomial $P_{\alpha}(z)=\frac{1}{\alpha 2^{\alpha}} \sum_{k=1}^{\alpha} \frac{\beta_{k}}{k}\left(z^{k}-1\right)$, where $\left(\beta_{k}\right)_{1 \leqq k<\alpha}$ satisfies (2.6), is a lower bound of the function $H(z)=\left(\frac{z+1}{2}\right)^{\alpha}$ In $z$, as well as an upper bound of the function $K(z)=\frac{z^{\alpha}-1}{\alpha}$.

Corollary 2.3: Suppose condition (2.6) holds. For $\mathrm{x}>\mathrm{y}>0$, the inequality

$$
\frac{x-y}{\operatorname{In} x-\operatorname{In} y}<\frac{\sum_{k=1}^{\alpha} \frac{\beta_{k}}{k}\left(x^{k / \alpha}-y^{k / \alpha}\right)}{2^{\alpha}(\operatorname{In} x-\operatorname{In} y)}<\left(\frac{x^{1 / \alpha}+y^{1 / \alpha}}{2}\right), \alpha \varepsilon \mathbb{Z}
$$

is valid whenever

$$
\begin{aligned}
x^{\frac{\alpha+1}{\alpha}} & +\sum_{k=2}^{\alpha}\left[{ }^{\alpha+1} C_{k}+\frac{\beta_{k-1}}{k-1}-\frac{\beta_{k-1}+\beta_{k}}{\alpha}\right] x^{k / \alpha}(\alpha+1-k) / \alpha \\
& +\left[\alpha+1-\frac{\beta_{1}}{\alpha}-\sum_{k=1}^{\alpha} \frac{\beta_{k}}{k}\right]=x^{1 / \alpha_{y}(\alpha+1) / \alpha}>0
\end{aligned}
$$

Proof: The result is immediate on setting $z^{\alpha}=\frac{x}{y}$, where $x>y>0$ in inequality (2.7). 
The cases $\alpha=2$ and $\alpha=3$ with $\beta_{k}=\alpha 2^{\alpha}$ and $\beta_{k}=0, k \neq \alpha$ lead to Carlson's result and Lin's inequality respectively. Suppose $\beta_{\alpha}=\alpha 2^{\alpha}(1+\xi / 2), \xi>0$; then inequality (2.8) gives an improved lower bound for the power mean $M_{1 / \alpha}$, subject to the condition (2.9). To see this, let us consider the following special cases.

Suppose $\alpha=1$. Then inequality (2.8) yields

$$
\frac{x-y}{\operatorname{In} x-\operatorname{In} y}<\left(1+\frac{\xi}{2}\right) \frac{x-y}{\operatorname{In} x-\operatorname{In} y}<\frac{x+y}{2}
$$

provided $\mathrm{x}>\mathrm{y}\left[1+\xi+\sqrt{ }\left(\xi^{2}+2 \xi\right)\right] ; \xi>0$

Suppose $\alpha=2$, we have

$$
\frac{x-y}{\operatorname{In} x-\operatorname{In} y}<\left(1+\frac{\xi}{2}\right) \frac{x-y}{\operatorname{In} x-\operatorname{In} y}<\left(\frac{x^{\frac{1}{2}}+y^{\frac{1}{2}}}{z}\right)^{2}
$$

wherever $\mathrm{x}>\mathrm{y}\left[1+\xi+\sqrt{ }\left(\xi^{2}+2 \xi\right)\right] ; \xi>0$.

If we finally consider the case when $\alpha=3$, then inequality (2.8) yields

$$
\frac{x-y}{\operatorname{In} x-\operatorname{In} y}<\left(1+\frac{\xi}{2}\right) \frac{x-y}{\operatorname{In} x-\operatorname{In} y}<\left(\frac{\left.x^{\frac{1}{3}}+y^{\frac{1}{3}}\right)^{3}}{2}\right.
$$

provided $x>y(1+\xi)+\sqrt{ }\left(\xi^{2}+2 \xi\right)+\sqrt{ }\left[2\left(\xi^{2}+2 \xi\right)+2(1+\xi) \sqrt{ }\left(\xi^{2}+2 \xi\right)\right] ; \quad \xi>0$.

We pointed out in Section 1 that inequality (1.3) holds for $p=\frac{1}{4}$ if $\frac{x}{y}>(9.9)^{4}$. It is pertinent to add here that these values can be obtained by solving for $\frac{x}{y}$ in the inequality (2.9) when $\alpha=4$. To illustrate the usefulness of this method, we compute the set on which inequality (1.3), with $p=\frac{1}{4}$, is true.

Take $\beta_{\alpha}=\alpha 2^{\alpha}, \beta_{k}=0, k \neq \alpha$ and $\alpha=4$ in inequality (2.8). The result is

$$
\frac{x-y}{\operatorname{In} x-\operatorname{In} y}<\left(\frac{x^{\frac{3}{4}}+y^{\frac{1}{4}}}{2}\right)
$$

provided $z^{5}-11 z^{4}+10 z^{3}+10 z^{2}-11 z+1>0$, where $z^{4}=x / y$. The left hand side of this inequality has the factorization $(z+1)(z-1)^{2}(z-5+2 \sqrt{6})(z-5-2 \sqrt{6})$. 
Consequently, inequality (2.13) is valid provided either $1>\left(\frac{x}{y}\right)^{\frac{1}{4}}>5-2 \sqrt{6}=0.10$ or $\left(\frac{x}{y}\right)^{\frac{1}{4}}>5+2 \sqrt{6}=9.9$. Since $x>y>0$, we have $L(x, y)<M_{\frac{1}{4}}(x, y)$ on the set $\left[(x, y) ; \frac{x}{y}>(9.9)^{4}\right]$. Similar results can be obtained for $\alpha=5,6,7, \ldots$

Further specializations of the constants $\beta_{k}, 1<k<\alpha$, may be of interest in the determination of improved upper and lower bounds for the logarithmic mean and the power mean respectively.

\section{$\underline{\text { REFERENCES }}$}

1. Tung-Po Lin: The Power Mean and the Logarithmic Mean; Amer. Math. Monthly 81 (1974), 879-883.

2. E.B. Leach and M.C. Sholander: Extended Mean Values; Amer. Math. Monthly $85(1978), 84-90$.

3. E.B. Leach and M.C. Sholander: Corrections to "Extended Mean Values"; Amer. Math. Monthly 85 (1978), 656.

4. K.B. Stolarsky: Generalizations of the Logarithmic Mean; Amer. Math. Mag. 48 (1975), 87-92.

5. K.B. Stolarsky: The Power and Generalized Logarithmic Mean; Amer. Math. Monthly, to appear.

6. B.C. Carlson: The Logarithmic Mean; Amer. Math. Monthly 79 (1972), 615-618. 


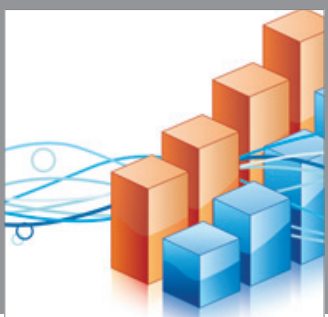

Advances in

Operations Research

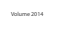

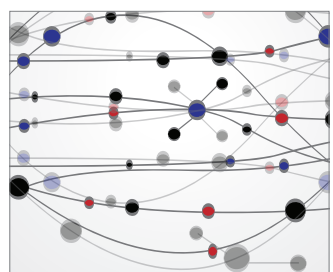

\section{The Scientific} World Journal
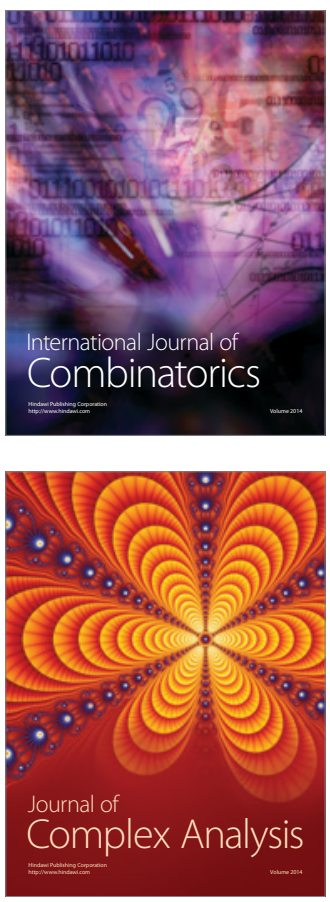

International Journal of

Mathematics and

Mathematical

Sciences
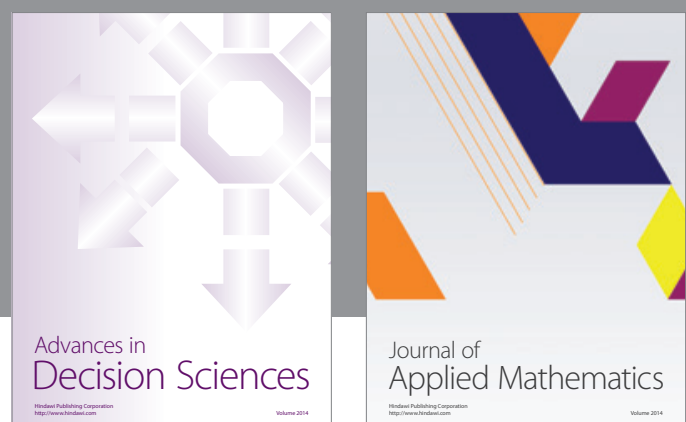

Journal of

Applied Mathematics
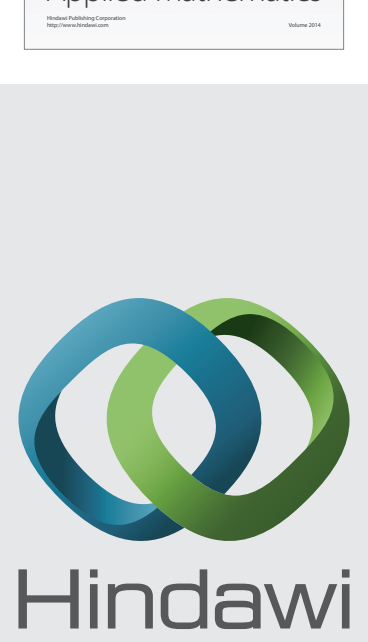

Submit your manuscripts at http://www.hindawi.com
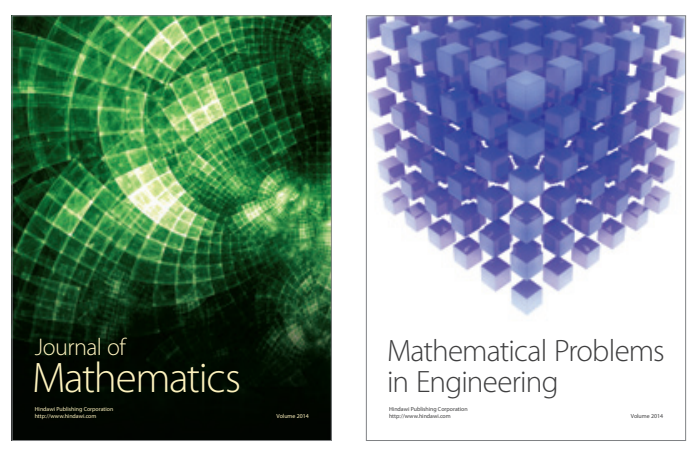

Mathematical Problems in Engineering
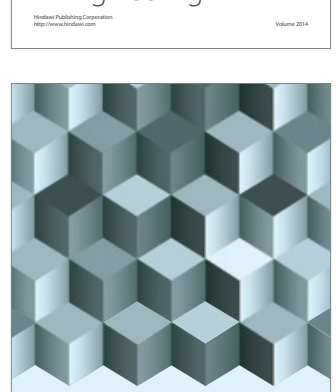

Journal of

Function Spaces
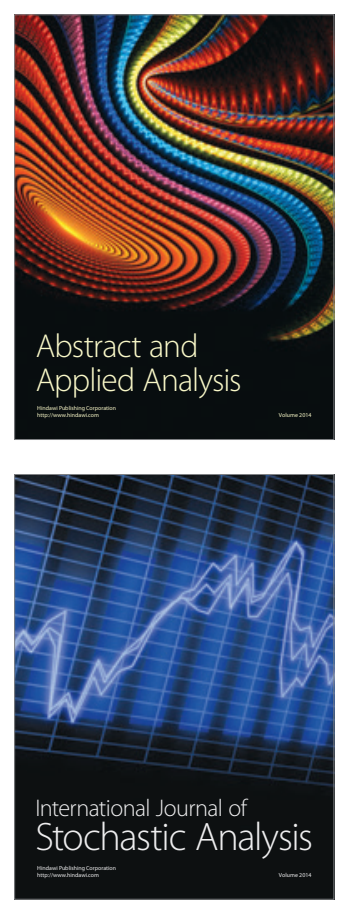

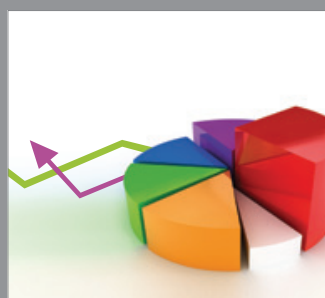

ournal of

Probability and Statistics

Promensencen
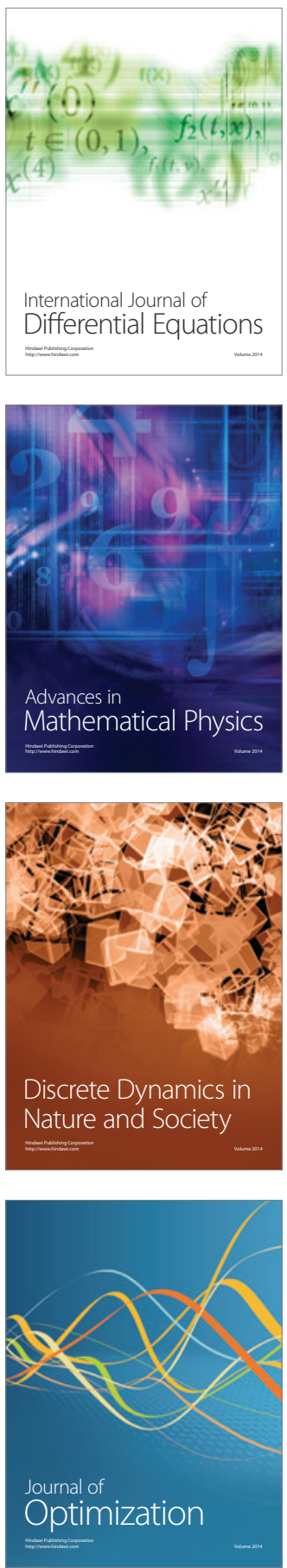